\title{
A STOCHASTIC COLLOCATION METHOD FOR STOCHASTIC VOLTERRA EQUATIONS OF THE SECOND KIND
}

\author{
YANZHAO CAO AND RAN ZHANG \\ Communicated by Terry Herdman
}

\begin{abstract}
This work describes and analyzes a stochastic collocation method for stochastic Volterra integral equations (SVIEs) of the second kind with random forcing terms. A collocation method is used in temporal direction, and a spectral collocation method is used in the stochastic dimension, which lead to an uncoupled linear system associated with the collocation points. The convergence analysis is carried out and the optimal error estimates are obtained. Numerical experiments are conducted to verify the error estimates and demonstrate the effectiveness of the proposed numerical method.
\end{abstract}

1. Introduction. Systems with uncertainties arise from the fact that uncertainties appear in most mathematical models of real world physical problems. The mathematical description of various processes in biological, physical and engineering sciences gives rise to stochastic or random differential equations and integral equations.

In this paper we are concerned with numerical approximations of the following SVIE of the second kind with a random forcing term.

$$
y(t, \omega)-\int_{0}^{t}(t-s)^{-\alpha} k(t, s) y(s, \omega) d s=g(t, \omega), \quad 0 \leq t \leq T, \omega \in \Omega
$$

2010 AMS Mathematics subject classification. Primary 65R20, 65Q20, 5D05.

Keywords and phrases. Stochastic collocation method, Volterra equation, random input data, spectral Galerkin method.

This work has been supported in part by NSF under grant number DMS0609918, by US Air Force Office for Scientific Research under grant numbers FA9550-121-0281, Guangdong Provincial Government of China through the Computational Science Innovative Research Team program. This work has been supported in part by NSFC $(11271157,11371171,11471141)$, and by the Program for New Century Excellent Talents in University of Ministry of Education of China. The second author is the corresponding author.

Received by the editors on July, 7, 2014. 
where $(\Omega, \mathcal{F}, P)$ is a complete probability space, $y=y(t, \omega)$ is an unknown random field whose value is to be determined on $[0, T] \times \Omega$, $k=k(t, s)$ is smooth on $[0, T] \times[0, T], 0 \leq \alpha<1$, and $g=g(t, \omega)$ is a random forcing term.

SVIEs can be derived from integral reformulations of stochastic partial differential equations with random initial values or forcing terms [29]. They can also be derived directly from many engineering and physical phenomena $[8,31,35]$. We refer to $[8,34,35]$ and the references therein for theoretical studies of SVIEs.

To solve SVIE (1) numerically the first step is to express the random forcing term $g=g(t, \omega)$ with a Fourier series like expansion. Two of the most commonly used methods for such an expansion are the polynomial chaos (PC) expansion [36] and the Karhunen-Loeve expansion (K-L) $[\mathbf{2 4}, \mathbf{2 5}]$. The PC expansion uses the orthogonal polynomial basis of independent random variables, while the K-L expansion represents the random quantity with an infinite number of uncorrelated random variables. Other nonlinear expansions have also been used to parameterize the input data (cf., $[\mathbf{1 3}, \mathbf{2 1}, \mathbf{2 8}, \mathbf{3 7}]$ ). Although both PC and K-L expansions are infinite series, in many practical applications, only a few terms in the expansions are needed to describe the randomness with desired accuracy. Therefore, we can assume that the expansion of $g$ only includes these terms. As a result, the exact solution $y$ of (1) depends on only a finite number of independent random variables which appear in the finite expansion of $g$.

The most commonly used numerical method for mathematical problems with random input is the Monte Carlo method (cf., [16] and references therein). The Monte Carlo method is easy to implement and allows one to reuse available deterministic codes. However, even with a mild dependence on the random variables, the convergence of the Monte Carlo simulation is still very slow. Recently, more efficient numerical methods for stochastic PDEs with random input data have been proposed. Among these, the spectral Galerkin method [6, 7, 18, 20], the stochastic collocation method $[3,5,4,27,32,38]$, perturbation methods or Neumann expansions have gained much attention $[\mathbf{1}, \mathbf{1 9}, \mathbf{3 3}, \mathbf{3 7}]$. It has been shown that spectral Galerkin/stochastic collocation methods based on the orthogonal tensor product polynomials feature a very fast convergence rate $[4,6,23]$. For instance, when 
the exact solution is analytic with respect to the random variables, a stochastic collocation method on the stochastic dimension was proved to converge exponentially with respect to the number of the collocation points employed for each random input variable [4].

The stochastic finite element method (cf., [15]), which employs standard finite element approximations in spatial domain and polynomial approximations in the probability domain, leads to a fully coupled system of linear equations requiring highly efficient strategies and parallel computations for its numerical solution. In comparison, the stochastic collocation method (cf., [4]) only requires collocating the equation at the zeros of tensor product orthogonal polynomials with respect to the joint probability density of the random variables. Stochastic collocation has already been applied in a variety of problems and is the subject of ongoing research $[\mathbf{5}, \mathbf{1 4}]$. One of the main advantages of the stochastic collocation method is that it leads to uncoupled deterministic problems. It can also treat efficiently the case of nonindependent random variables with the introduction of an auxiliary density.

Most of the aforementioned references focus on stochastic partial differential equations. We refer to $[\mathbf{2 2 , 2 6}$ ] for some recent work on numerical approximations of stochastic integral equations. In this paper, we will describe and analyze the stochastic collocation method for SVIE (1). As in the SPDEs cases, the stochastic collocation method for the SVIE naturally leads to uncouple deterministic problems. The main difference in the numerical treatment between the stochastic collocation method for SPDEs and the stochastic collocation method for SVIEs studied in this paper is that, unlike the SPDE cases, we use the collocation method on both physical (temporal) domains and probability domains, which further reduces the computing cost. Our work includes both error estimates and numerical experiments. The first step of deriving the error estimate is to obtain an error estimate for the semi-discrete collocation approximation in the temporal direction which must be uniform with respect to the random variables. As shown in our error analysis, the singularity expansion of the exact solution $[\mathbf{9}, \mathbf{1 1}, \mathbf{1 2}]$ turns out to be an ideal tool for such a purpose.

The paper is organized as follows. In Section 2, we describe the parameterization of SVIE (1) and its stochastic spectral collocation approximation. In Section 3, we prove the convergence and obtain the rate of convergence for the stochastic collocation approximation of (1). 
Finally, in Section 4, we verify our theoretical results and demonstrate the efficiency of our numerical algorithm with numerical experiments.

\section{The stochastic collocation method.}

2.1. Parameterization in stochastic dimensions. Let $(\Omega, \mathcal{F}, P)$ be a complete probability space and $\zeta: \Omega \rightarrow \mathbb{R}$ a real-valued random variable such that $\zeta \in L^{1}(\Omega)$. The mean or expected value of $\zeta$ is defined as

$$
E[\zeta]=\int_{\Omega} \zeta(\omega) d P(\omega) .
$$

Introduce function spaces

$$
L^{2}(\Omega):=\left\{v ; \int_{\Omega} v^{2}(\omega) d P(\omega)<\infty\right\}
$$

and

(2)

$$
V_{L^{2}([0, T]) \otimes L^{2}(\Omega)}:=\left\{v ; \int_{0}^{T} E\left[v^{2}\right] d s=\int_{0}^{T} \int_{\Omega} v^{2}(s, \omega) d P(\omega) d s<\infty\right\} .
$$

In what follows, we assume that

(A1) (finite dimensional noise) the forcing term $g$ has the form

$$
g(t, \omega)=f\left(t, Y_{1}(\omega), \cdots, Y_{N}(\omega)\right) \quad \text { on }[0, T] \times \Omega,
$$

where $N \in \mathbb{N}^{+}$and $\left\{Y_{n}\right\}_{n=1}^{N}$ are real-valued random variables with zero mean value and unit variance.

We denote by $\Gamma_{n}:=Y_{n}(\Omega)$ the image of $Y_{n}$ and $\Gamma:=\bigotimes_{n=1}^{N} \Gamma_{n}$. For simplicity of the presentation, we assume that $\Gamma$ is a bounded open box in $\mathcal{R}^{N}$. We also assume that $\left[Y_{1}, Y_{2}, \ldots, Y_{N}\right]$ has a joint probability density function

$$
\rho: \Gamma \longrightarrow R^{+}, \quad \text { with } \rho \in L^{\infty}(\Gamma) .
$$

By assumption (A1), the solution $y=y(t, \omega)$ of the problem (1) can be described by a finite number of independent random variables, that is,

$$
y(t, \omega)=y\left(t, Y_{1}(\omega), \cdots, Y_{N}(\omega)\right) .
$$


Then SVIE (1) becomes finding $y \in V_{L^{2}([0, T]) \otimes L_{\rho}^{2}(\Gamma)}$ such that

(4) $y(t, \theta)-\int_{0}^{t}(t-s)^{-\alpha} k(\tau, s) y(s, \theta) d s=g(t, \theta), \quad 0<t<T, \theta \in \Gamma$,

where the space $V_{L^{2}([0, T]) \otimes L_{\rho}^{2}(\Gamma)}$ is the analogue of $V_{L^{2}([0, T]) \otimes L^{2}(\Omega)}$ with $(\Omega, \mathcal{F}, P)$ replaced by $\left(\Gamma, \mathcal{B}^{N}, \rho d \theta\right)$. Thus, the stochastic Volterra equation problem (1) now becomes a deterministic Volterra problem with an $N$-dimensional parameter. For convenience, we use the notation $y(\theta)$ whenever we emphasize the dependence of $y$ on the parameter $\theta$.

2.2. Stochastic collocation method. The goal of the stochastic collocation method is to seek a numerical approximation to the exact solution of (4) in a finite dimensional subspace $V_{m, p}$ based on a tensor product $V_{m, p}=S_{m}([0, T]) \otimes \mathcal{P}_{p}(\Gamma)$. We assume that $\left(C_{1}\right) S_{m}([0, T]) \subset$ $L^{2}([0, T])$, is the space of piecewise polynomials of degree $m$ with respect to a partition on temporal direction (with dimension $N_{m}$ )

$$
0=t_{0}<t_{1}<\cdots<t_{M}=T
$$

$\left(C_{2}\right) t_{i j}, j=1, \ldots m+1, i=1, \ldots M$ are the collocation points defined as

$$
t_{i j}=t_{i-1}+c_{j}\left(t_{i}-t_{i-1}\right)
$$

where $0<c_{1}<c_{2}<\cdots<c_{m+1}<1$ are constants;

$\left(C_{3}\right) \mathcal{P}_{p}(\Gamma) \subset L_{\rho}^{2}(\Gamma)$ is the span of tensor product polynomials with degree at most $p=\left(p_{1}, \cdots, p_{N}\right)$, i.e., $\mathcal{P}_{p}(\Gamma)=\otimes_{n=1}^{N} \mathcal{P}_{p_{n}}\left(\Gamma_{n}\right)$, with

$$
\mathcal{P}_{p_{n}}\left(\Gamma_{n}\right)=\operatorname{span}\left(y_{n}^{j}, j=0, \cdots, p_{n}\right), \quad n=1, \ldots, N .
$$

Hence, the dimension of $\mathcal{P}_{p}$ is $N_{p}=\prod_{n=1}^{N}\left(p_{n}+1\right)$.

To construct the fully discrete stochastic collocation approximation, we first introduce the semidiscrete collocation approximation $y_{m}(\theta)=$ $y_{m}(t, \theta) \in S_{m}([0, T])$ in temporal direction as follows. For $\theta \in \Gamma$ and $j=1, \ldots, m+1, i=1, \ldots, M$,

$$
\begin{aligned}
& y_{m}\left(t_{i j}, \theta\right)-\int_{t_{i-1}}^{t_{i j}}\left(t_{i j}-s\right)^{-\alpha} k\left(t_{i j}, s\right) y_{m}(s, \theta) d s \\
& =\int_{0}^{t_{i-1}}\left(t_{i-1}-s\right)^{-\alpha} k\left(t_{i-1}, s\right) y_{m}(s, \theta) d s+g\left(t_{i j}, \theta\right) .
\end{aligned}
$$


Next we collocate (6) on the zeros of Legendre or Hermite orthogonal polynomials and build the approximate solution $y_{m, p} \in S_{m}([0, T]) \otimes$ $\mathcal{P}_{p}(\Gamma)$ by interpolating in $\theta$ the collocated solutions. To this end, we first introduce an auxiliary probability density function $\widehat{\rho}: \Gamma \rightarrow \mathbb{R}^{+}$ that can be seen as the joint probability of $N$ independent random variables, i.e., it factorizes as

(7) $\widehat{\rho}(\theta)=\prod_{n=1}^{N} \widehat{\rho}_{n}\left(\theta_{n}\right), \quad$ for all $\theta \in \Gamma$, and such that $\left\|\frac{\rho}{\widehat{\hat{\rho}}}\right\|_{L^{\infty}(\Gamma)}<\infty$.

For each $n=1, \ldots, N$, let $\theta_{n, k_{n}}\left(1 \leq k_{n} \leq p_{n}+1\right)$ be the $p_{n}+1$ roots of the orthogonal polynomial $q_{p_{n}+1}$ according to the weight $\hat{\rho}_{n}$, which then satisfies

$$
\int_{\Gamma_{n}} q_{p_{n}+1}(\theta) v(\theta) \hat{\rho}_{n}(\theta) d \theta=0
$$

for all $v \in \mathcal{P}_{p_{n}}\left(\Gamma_{n}\right)$.

We denote by $\theta_{k}$ the point $\theta_{k}=\left[\theta_{1, k_{1}}, \theta_{2, k_{2}}, \ldots, \theta_{N, k_{N}}\right] \in \Gamma$ and introduce, for $n=1,2, \ldots, N$, the Lagrange basis $\left\{l_{n, j}\right\}_{j=1}^{p_{n}+1}$ of the space $\mathcal{P}_{p_{n}}$ such that

$$
l_{n, j} \in \mathcal{P}_{p_{n}}\left(\Gamma_{n}\right), \quad l_{n, j}\left(\theta_{n, k}\right)=\delta_{j k}, \quad j, k=1, \ldots, p_{n}+1,
$$

where $\delta_{j k}$ is the Kronecker symbol. Let $l_{k}(\theta)=\prod_{n=1}^{N} l_{n, k_{n}}\left(\theta_{n}\right)$. Then the collocation approximation is defined as

$$
y_{m, p}(t, \theta)=\sum_{k=1}^{N_{p}} y_{m}\left(t, \theta_{k}\right) l_{k}(\theta),
$$

where $y_{m}\left(t, \theta_{k}\right)$ is the solution of problem (6) for $\theta=\theta_{k}$. Define the Lagrange interpolation operator $\mathcal{I}_{p}: C^{0}\left(\Gamma ; S_{m}([0, T])\right) \rightarrow S_{m}([0, T]) \otimes$ $\mathcal{P}_{p}(\Gamma)$, such that

$$
\mathcal{I}_{p} v(\theta)=\sum_{k=1}^{N} v\left(\theta_{k}\right) l_{k}(\theta), \quad \text { for all } v \in C^{0}\left(\Gamma ; S_{m}([0, T])\right) .
$$

We can now express $y_{m, p}$ as $y_{m, p}=\mathcal{I}_{p} y_{m}$.

3. Convergence analysis. In this section we study the convergence of the stochastic collocation scheme proposed in the last section. First 
we introduce the function space

(11)

$C^{0}(\Gamma ; B):=\left\{v: \Gamma \rightarrow B, v\right.$ is continuous in $\left.\theta, \max _{\theta \in \Gamma}\|v(\theta)\|_{B}<\infty\right\}$,

where $B$ is a Banach space. We assume that

(A2) $g \in C^{0}\left(\Gamma ; L^{2}([0, T])\right)$, and

(A3) the joint probability density $\rho$ satisfies the following inequality

$$
\rho(\theta) \leq C_{\rho}
$$

where $C_{\rho}$ is a constant independent of $\theta$.

Next we choose a suitable auxiliary density $\widehat{\rho}(\theta)=\prod_{k=1}^{N} \widehat{\rho}_{n}\left(\theta_{n}\right)$ which satisfies

$$
C_{1}^{n} \leq \widehat{\rho}_{n}\left(\theta_{n}\right) \leq C_{2}^{n}, \quad \text { for all } \theta_{n} \in \Gamma_{n}, \quad n=1, \ldots, N,
$$

for some positive constants $C_{1}^{n}$ and $C_{2}^{n}$ that do not depend on $\theta_{n}$. We remark that, under this assumption, $\widehat{\rho}$ satisfies (7).

Before presenting the convergence analysis, we need a result on the analyticity of the exact solution $u$ with respect to $\theta$. Let $K$ be the linear operator on $L^{2}([0, T])$ such that, for $u \in L^{2}([0, T])$,

$$
(K u)(t)=\int_{0}^{t}(t-s)^{-\alpha} k(t, s) u(s, \omega) d s, \quad 0 \leq t \leq T .
$$

Then we can rewrite (1) as

$$
(I-K) y(t, \theta)=g(t, \theta), \quad 0<t<T, \theta \in \Gamma .
$$

Assume that 1 is not an eigenvalue of $K$. Since $K$ is compact, $(I-K)^{-1}$ exists and is continuous.

The following lemma concerns the analyticity of the exact solution $y$ with respect to $\theta$.

Lemma 3.1. Denote

$$
\Gamma_{n}^{*}=\prod_{j=1, j \neq n}^{N} \Gamma_{j}
$$


and

$$
\widehat{\rho}_{n}^{*}=\prod_{j=1, j \neq n}^{N} \widehat{\rho}_{j} .
$$

Assume that, for every $\theta=\left(\theta_{n}, \theta_{n}^{*}\right) \in \Gamma$, there exists $\gamma_{n}<+\infty$ such that

$$
\left\|\partial_{\theta_{n}}^{k} g(\theta)\right\|_{L^{2}([0, T])} \leq k ! \gamma_{n}^{k}
$$

Then the solution $y=y\left(t, \theta_{n}, \theta_{n}^{*}\right)$ as a function of $\theta_{n}, y: \Gamma_{n} \rightarrow$ $C^{0}\left(\Gamma_{n}^{*} ; L^{2}([0, T])\right)$ admits an analytic extension $y\left(t, z, \theta_{n}^{*}\right), z \in \mathbb{C}$, in the region of the complex plane

$$
\Sigma\left(\Gamma_{n} ; \tau_{n}\right):=\left\{z \in \mathbb{C}, \operatorname{dist}\left(z, \Gamma_{n}\right) \leq \tau_{n}\right\}
$$

with $0<\tau_{n}<\gamma_{n}$.

Proof. From (13), the $k$ th derivative of $y$ with respect to $\theta_{n}$ satisfies

$$
(I-K) \partial_{\theta_{n}}^{k} y(t, \theta)=\partial_{\theta_{n}}^{k} g(t, \theta), \quad 0<t<T, \theta \in \Gamma .
$$

Thus,

$$
\left\|\partial_{\theta_{n}}^{k} y(\cdot, \theta)\right\|_{L^{2}([0, T])} \leq\left\|(I-K)^{-1}\right\|\left\|\partial_{\theta_{n}}^{k} g(\cdot, \theta)\right\|_{L^{2}([0, T])} .
$$

Define for every $\theta_{n} \in \Gamma_{n}$ the power series $y: \mathbb{C} \rightarrow C^{0}\left(\Gamma_{n}^{*}, L^{2}(\Omega)\right)$ as

$$
y\left(t, z, \theta^{*}\right)=\sum_{k=0}^{\infty} \frac{\left(z-\theta_{n}\right)^{k}}{k !} \partial_{\theta_{n}}^{k} y\left(t, \theta_{n}, \theta_{n}^{*}\right) .
$$

Then, from (14) and (16), we have that

$$
\begin{aligned}
& \|y(t, z, \cdot)\|_{C^{0}\left(\Gamma_{n}^{*}, L^{2}(\Omega)\right)} \\
& \leq\left\|(I-K)^{-1}\right\| \sum_{k=0}^{\infty} \frac{\left|z-\theta_{n}\right|^{k}}{k !}\left\|\partial_{\theta_{n}}^{k} y\left(t, \theta_{n}, \cdot\right)\right\|_{C^{0}\left(\Gamma_{n}^{*}, L^{2}(\Omega)\right)} \\
& \leq\left\|(I-K)^{-1}\right\| \sum_{k=0}^{\infty}\left(\left|z-\theta_{n}\right| \gamma_{n}\right)^{k}
\end{aligned}
$$

Therefore, the series converges for all $z \in \mathbb{C}$ such that $\left|z-\theta_{n}\right|<$ $\tau_{n}<\gamma_{n}$. By a continuation argument, the function $y$ can be extended analytically on the whole region $\Sigma\left(\Gamma_{n}, \tau_{n}\right)$. 
The next lemma gives an error estimate of the semi-discrete approximation $y_{m}$.

Lemma 3.2. Assume that

(i) $k \in C^{m+1}([0, T] \times[0, T])$ and $g \in C^{0}\left(\Gamma, C^{m+1}([0, T])\right)$ in (1);

(ii) the graded partition of $[0, T]$ is given by

$$
t_{i}=\left(\frac{i}{M}\right)^{r} T, \quad i=0, \ldots M,
$$

where $r(\alpha)=(m+1) /(1-\alpha)$. Then there exists a positive constant $\bar{h}$ such that

$$
\begin{aligned}
& \left\|y-y_{m}\right\|_{C^{0}\left(\Gamma, L_{\infty}([0, T])\right)} \\
& \quad \leq C\left(T, m,\left\{c_{i}\right\}, \alpha\right)\|g\|_{C^{0}\left(\Gamma, C^{m+1}([0, T])\right)} h^{m+1}
\end{aligned}
$$

holds for any $h=T / M \in(0, \bar{h})$ and collocation point set $\left\{c_{j}\right\}_{j=1}^{m+1}$.

Proof. Using [9, (6.1.17)] (see also [11, (2.1)] and [12, (2.1)]) we have that

$$
y(t)=\sum_{(j, k)_{\alpha}} \chi_{j, k}(g) t^{j+k(1-\alpha)}+u_{m}(t, \theta), \quad t \in[0, T], \theta \in \Gamma,
$$

where

$$
(j, k)_{\alpha}=\left\{(j, k), \quad j, k \in \mathbb{N}_{0}, j+k(1-\alpha)<m+1\right\},
$$

$u_{m} \in C^{0}\left(\Gamma, C^{m+1}([0, T])\right)$ and $\chi_{j, k}(g)$ are constants depending on $g$. Using the resolvent formula $(6.1 .11)$ of [9] we deduce that $\chi_{j, k}(\cdot)$ are linear functionals defined on $C^{0}\left(\Gamma, C^{m+1}([0, T])\right)$, i.e., there exist constants $C_{j, k}$ such that

$$
\left|\chi_{j, k}(g)\right| \leq C_{j, k}\|g\|_{C^{0}\left(\Gamma, C^{m+1}([0, T])\right)} .
$$

Using the above estimate and following the proof of Theorem 6.1.4 of [9] on the global error estimate (cf., Appendix), we derive that

$$
\left\|y-y_{m}\right\|_{C^{0}\left(\Gamma, L_{\infty}([0, T])\right)} \leq C\left(T, m,\left\{c_{i}\right\}, \alpha\right)\|g\|_{C^{0}\left(\Gamma, C^{m+1}([0, T])\right)} h^{m+1} .
$$

Lemma $3.3([4])$. Under the assumptions of Lemma 3.1, there exist positive constants $C$ and $r_{n}, n=1, \ldots, N$, independent of $m$ and $p$, 
such that

$$
\left\|y_{m}-y_{m, p}\right\|_{L^{2}([0, T]) \otimes L_{\rho}^{2}(\Gamma)} \leq C \sum_{n=1}^{N} \beta_{n}\left(p_{n}\right) \exp \left\{-r_{n} p_{n}^{\theta_{n}}\right\},
$$

where

$$
r_{n}=\log \left[\frac{2 \tau_{n}}{\left|\Gamma_{n}\right|}\left(1+\sqrt{1+\frac{\left|\Gamma_{n}\right|^{2}}{4 \tau_{n}^{2}}}\right)\right] .
$$

Here $\tau_{n}$ is defined in Lemma 3.1.

We are now ready to give the error estimates for the numerical solution $y_{m, p}$ to SVIE (1).

Theorem 3.4. Under the assumptions of Lemma 3.1 and Lemma 3.2, there exist positive constants $C$ and $r_{n}, n=1, \ldots, N$, which are independent of $m$ and $p$, such that

$$
\left\|y-y_{m, p}\right\|_{L^{2}([0, T]) \otimes L_{\rho}^{2}(\Gamma)} \leq C\left(h^{m+1}+\sum_{n=1}^{N} \beta_{n}\left(p_{n}\right) \exp \left\{-r_{n} p_{n}^{\theta_{n}}\right\}\right)
$$

where the parameters $\theta_{n}, \beta_{n}$ and $r_{n}$ are specified in Lemma 3.3.

Proof. By the construction of $y_{m, p}$ and the triangular inequality, we have

$$
\begin{aligned}
\left\|y-y_{m, p}\right\|_{L^{2}([0, T]) \otimes L_{\rho}^{2}(\Gamma) \leq} & \left\|y-y_{m}\right\|_{L^{2}([0, T]) \otimes L_{\rho}^{2}(\Gamma)} \\
& +\left\|y_{m}-y_{m, p}\right\|_{L^{2}([0, T]) \otimes L_{\rho}^{2}(\Gamma)} .
\end{aligned}
$$

The first term on the right-hand side of (20) only depends on the temporal discretization. By Lemma 3.2, we have that

$$
\begin{aligned}
\left\|y-y_{m}\right\|_{L^{2}([0, T]) \otimes L_{\rho}^{2}(\Gamma)} & =\left(\int_{\Gamma} \int_{0}^{T}\left|y(\tau)-y_{m}(\tau)\right|^{2} \rho(\theta) d \tau d \theta\right)^{1 / 2} \\
& \leq\left\|y-y_{m}\right\|_{\infty} \int_{\Gamma} \int_{0}^{T} \rho(\theta) d \tau d \theta \\
& =T\left\|y-y_{m}\right\|_{\infty} \\
& \leq C h^{m+1}
\end{aligned}
$$


The second term is the interpolation error between the semidiscrete solution $y_{m}$ and the full discrete solution $y_{m, p}$, whose error is given by (19) in Lemma 3.3. Combining these two error estimates, we obtain the desired result.

Using the above error estimates and following arguments similar to those in Lemma 4.7 and Lemma 4.8 of [4], we have the following error estimates for the first two moments.

Proposition 3.5. The following hold

$$
\begin{aligned}
\left\|E\left[y-y_{m, p}\right]\right\|_{L^{2}([0, T])} & \leq C\left\|y-y_{m, p}\right\|_{L^{2}([0, T]) \otimes L_{\rho}^{2}(\Omega)}, \\
\left\|E\left[y^{2}-y_{m, p}^{2}\right]\right\|_{L^{1}\left(D_{R_{2}}\right)} & \leq C\left\|y-y_{m, p}\right\|_{L^{2}([0, T]) \otimes L_{\rho}^{2}(\Omega)},
\end{aligned}
$$

with $C$ independent of the discretization parameters $h$ and $p$.

4. Numerical experiments. In this section, we carry out numerical experiments to verify the convergence results derived in the last section and demonstrate the effectiveness of the proposed numerical algorithm.

Example 4.1. In this example, we consider the stochastic Volterra integral equation

$$
y(t, \omega)-\frac{1}{2} \int_{0}^{t}(t-s)^{2} e^{s-t} y(s, \omega) d s=g(t, \omega), \quad 0 \leq t \leq 1,
$$

with the forcing term

$$
\begin{aligned}
g(t, \omega)= & \frac{1}{2} t^{2} e^{-t}+\exp \left\{\left[Y_{1}(\omega) \cos \pi t+Y_{2}(\omega) \sin \pi t\right.\right. \\
& \left.\left.+Y_{3}(\omega) \cos 2 \pi t+Y_{4}(\omega) \sin 2 \pi t\right] e^{-1 / 8}\right\} .
\end{aligned}
$$

Here, the random variables $Y_{n}, n=1,2,3,4$, are independent and identically distributed (iid) with zero mean and unit variance. The exact solution is given by

$$
y(t)=\frac{1}{3}\left[1-e^{-(3 t) / 2}\left(\cos \left(\frac{\sqrt{3} t}{2}\right)+\sqrt{3} \sin \left(\frac{\sqrt{3} t}{2}\right)\right)\right]
$$

in the absence of the noise forcing term. In accordance with Theorem 3.4, we choose the finite dimensional space for temporal direction 
as the space of piecewise polynomials of degree three with $M=1000$ on $[0,1]$. With such a large $M$, the overall numerical error will be dominated by the error of the collocation approximation in the stochastic dimension. Thus we can verify the exponential decrease of the collocation errors in the stochastic dimension as the polynomial order of the collocation interpolation increases. We consider two different cases, the first case assumes uniform distribution for $Y_{n}$ and the second case assumes Gaussian densities. The corresponding collocation points are the roots of either Legendre or Hermite polynomials.
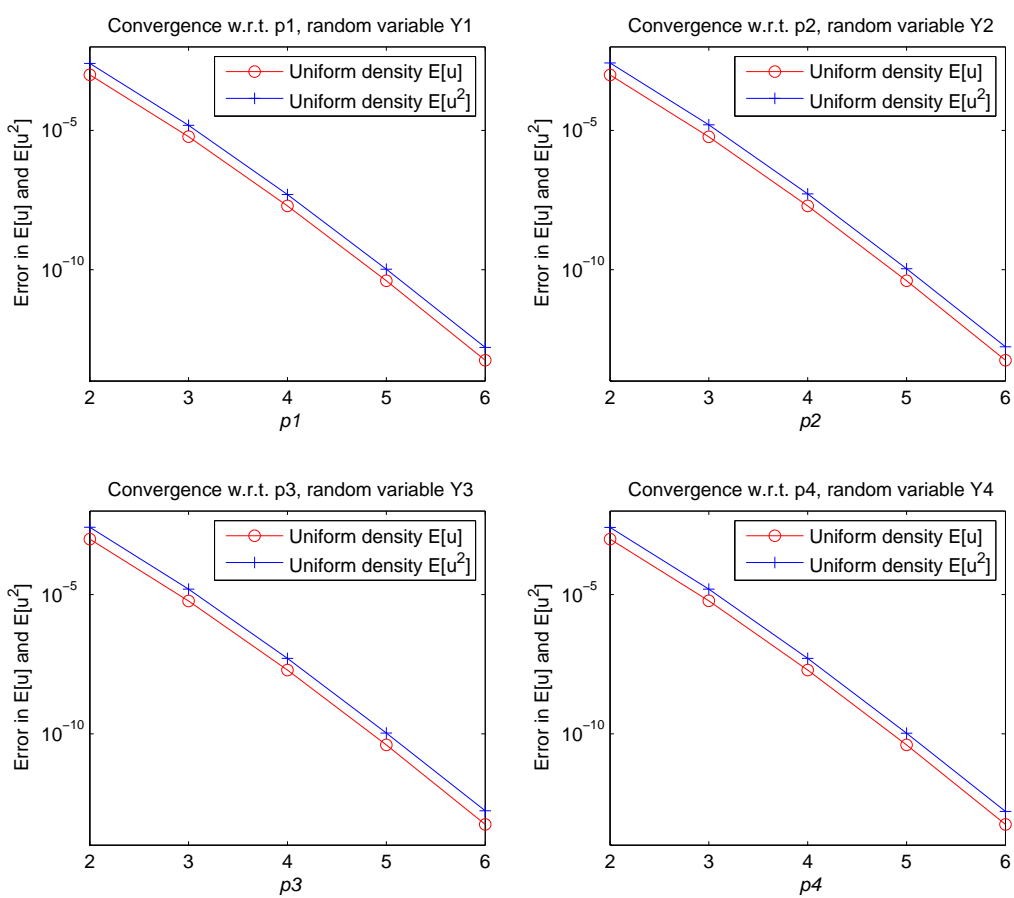

FiguRE 1. Convergence results for the approximation of expected value and second moment with uniform density in Example 4.1.

The computational results for the $L^{2}([0,1])$ approximation error in the expected value and the second moment are shown in Figure 1 with 

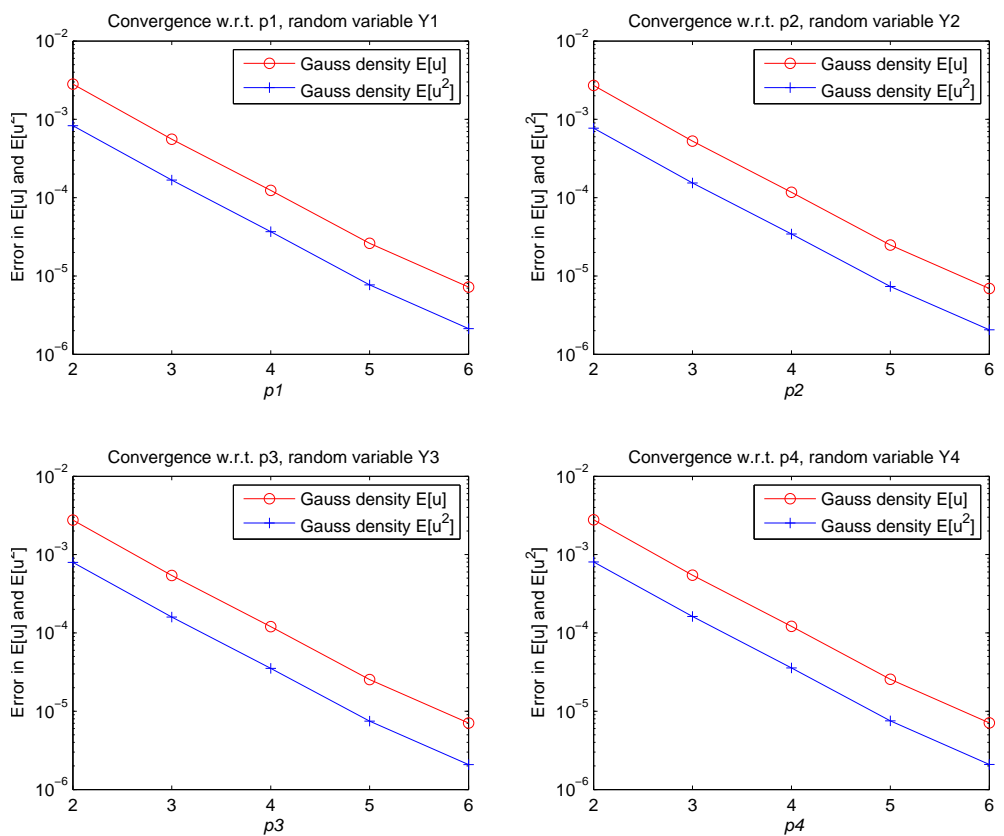

FiguRE 2. Convergence results for the approximation of expected value and second moment with Gauss density in Example 4.1.

uniform density, while the corresponding results are shown in Figure 2 with Gauss density. In Figure 1, the vertical axis denotes the expectation errors between numerical solution and exact solution (the dot-line and plus-line denote $\mathrm{E}(\mathrm{u})$ and $\mathrm{E}\left(u^{2}\right)$ respectively), and the horizontal axis denotes the degree of polynomial in certain direction $p_{i}$. The four sub-figures denote the error for different directions $p_{1}, p_{2}, p_{3}$ and $p_{4}$, and we use similar notation for the rest of the figures. To estimate the computational error in the $i$ th direction corresponding to a multi-index $p=\left(p_{1}, \ldots, p_{i}, \ldots, p_{N}\right)$, we approximate it by $E[e] \approx E\left[y_{m, p}-y_{m, \widehat{p}}\right]$, with $\widehat{p}=\left(p_{1}, \ldots, p_{i}+1, \ldots, p_{N}\right)$. We proceed similarly for the error in the approximation of the second moment. As expected, the estimated approximation error decreases exponentially as the polynomial degree increases. As shown in [4], these results indicate the exponential decay of the errors with respect to the degree of the polynomials used in the 

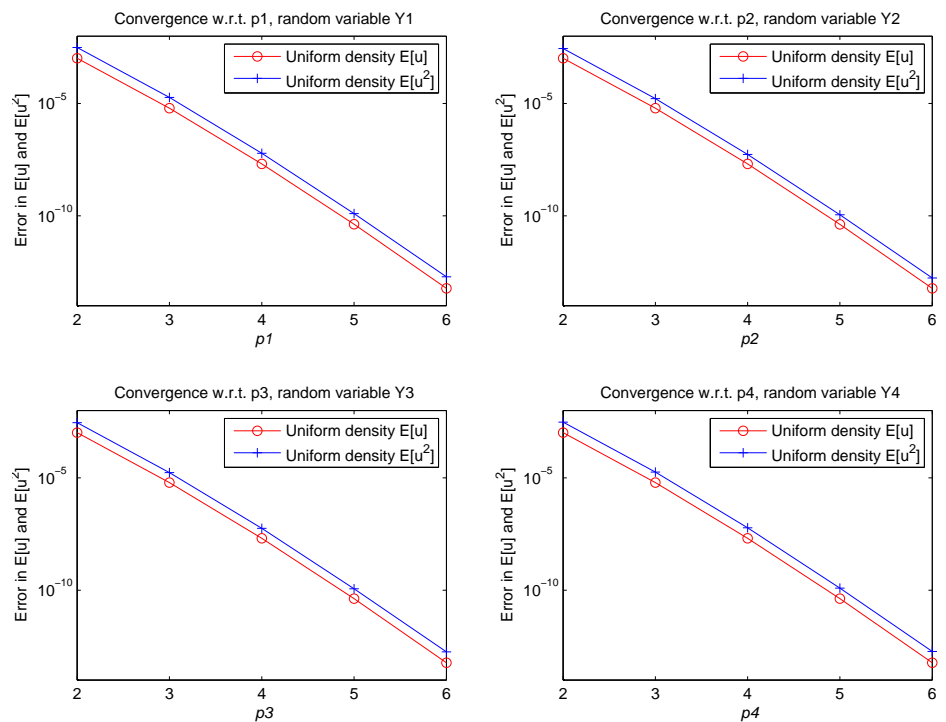

FIGURE 3. Convergence results for the approximation of expected value and second moment with uniform density in Example 4.2.

spectral collocation approximation.

Example 4.2. In this example, we consider the following test problem

$$
y(t, \omega)=\frac{1}{2} \int_{0}^{t}(t-s)^{-1 / 2} y(s, \omega) d s+g(t, \omega), \quad t \in(0, T]
$$

with the forcing term

$$
\begin{aligned}
g(t, \omega)= & -\frac{1}{2} \pi t+\sqrt{t}+\exp \left\{\left[Y_{1}(\omega) \cos \pi t+Y_{2}(\omega) \sin \pi t\right.\right. \\
& \left.\left.+Y_{3}(\omega) \cos 2 \pi t+Y_{4}(\omega) \sin 2 \pi t\right] e^{-1 / 8}\right\} .
\end{aligned}
$$

Here, the real random variables $Y_{n}, n=1, \ldots, 4$ are iid with zero 

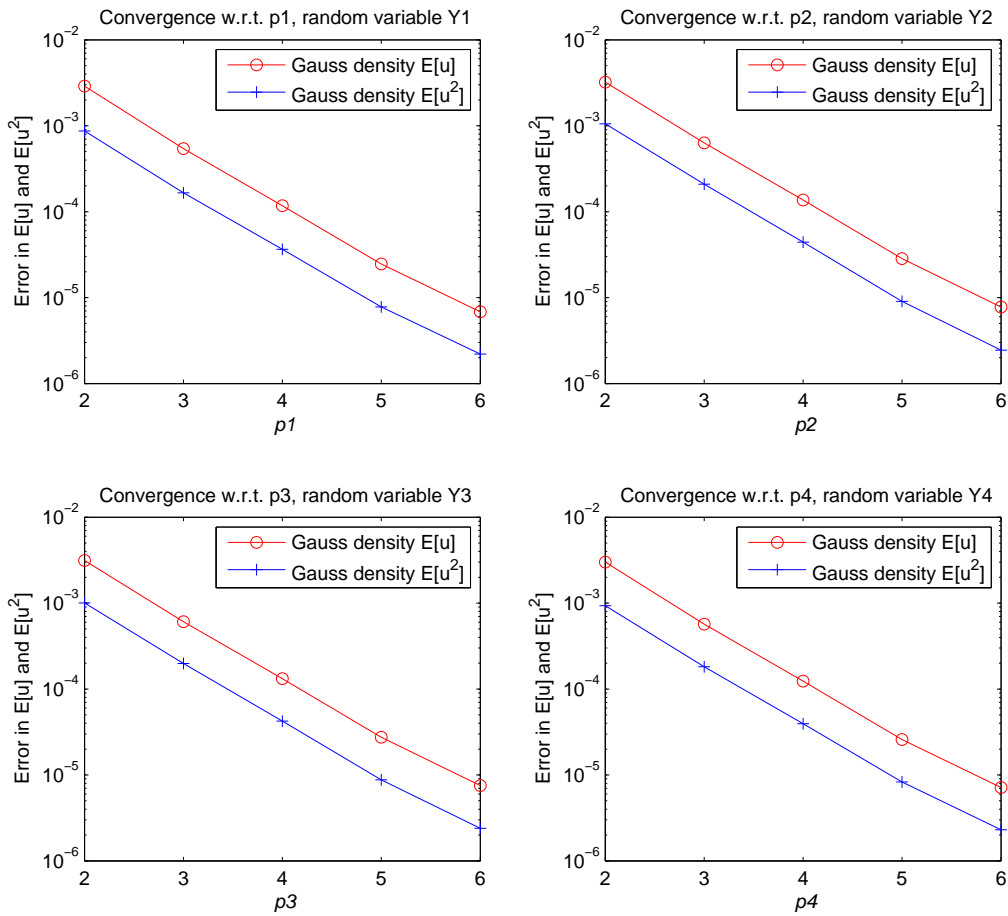

FIGURE 4. Convergence results for the approximation of expected value and second moment with Gauss density in Example 4.2.

expectation and unit variance, and the exact solution is

$$
y(t)=\sqrt{t}
$$

in the absence of the noise forcing term. Since the kernel has weak singularity, we use the geometric partition in the temporal direction given by (17) with $r=1 / 2$.

In our numerical simulation, we choose $T=1, K=1000, k=$ $1 / 1000, n=14$ and $\mu=0.5$, i.e., the partition is given by

$$
t_{0}=0, \quad t_{m}=\left(\frac{1}{2}\right)^{15-m} k, \quad 1 \leq m \leq 15
$$




$$
t_{m}=(m-15) k, \quad 17 \leq m \leq 1014 .
$$

Similar to the first example, we choose the finite dimensional space for the temporal approximation to be the piecewise polynomials of degree three on the above given partition, which again makes the collocation error in stochastic dimension dominate the overall numerical error. The computational results for the $L^{2}([0,1])$ approximation error in the expected value and the second moment are shown in Figure 3 with uniform density, while the corresponding results are shown in Figure 4 with Gauss density. Once again, the graphs show the estimated approximation error decreases exponentially as the polynomial degree increases.

5. Conclusions and future work. In this paper, we describe and analyze a stochastic collocation method for Volterra integral equations of the second kind with random forcing terms. The input data are assumed to depend on a finite number of random variables. We use the collocation method in both the temporal direction and the probability space and provide a complete convergence analysis. Our analysis shows the exponential convergence of the collocation method under the analyticity assumption for the forcing term with respect to the parameterized random variables. In future research, we plan to apply the stochastic collocation method to the stochastic Volterra integral equations with delays, which have many applications in practice. Future research also includes the stochastic collocation method for stochastic fractional differential equations that can be converted to stochastic Voltera integral equations (see [17]).

Acknowledgments. The authors thank the anonymous referees and the Associate Editor for their careful reading of the manuscript and their valuable comments.

\section{APPENDIX}

The purpose of this appendix is to fill the details of the proof of Lemma 3.2. To this end, we need the following technical lemma whose proof is straightforward.

Lemma 5.1. Let $I_{h}$ be the graded mesh of Lemma 3.2 on $I=[0, T]$ with grading exponent $r \geq 1$. If the $\left\{c_{i}\right\}$ satisfy $0 \leq c_{1}<\cdots<c_{m} \leq 1$, 
then for $1 \leq \ell<n \leq N-1$ and $\nu \in \mathrm{N}_{0}$,

$$
\int_{0}^{1}\left(\frac{t_{n, i}-t_{\ell}}{h_{\ell}}-s\right)^{-\alpha} s^{\nu} d s \leq \gamma(\alpha)(n-\ell)^{-\alpha} \quad(i=1, \ldots, m)
$$

with $\gamma(\alpha):=2^{\alpha} /(1-\alpha)$.

Proof of global error estimate in Lemma 3.2. The proof follows closely the proof of Theorem 2.2.1 of [9], except that now the local (Peano) representation of the exact solution $y$ on $\bar{\sigma}_{n}$ remains valid only if $n=1, \ldots, N-1$. The collocation error $e_{h}:=y-y_{m}$ satisfies the error equation

$$
\begin{aligned}
e_{h}\left(t_{n, i}\right)= & \int_{0}^{t_{1}} H_{\alpha}\left(t_{n, i}, s\right) e_{h}(s) d s+\int_{t_{1}}^{t_{n}} H_{\alpha}\left(t_{n, i}, s\right) e_{h}(s) d s \\
& +h_{n} \int_{0}^{c_{i}} H_{\alpha}\left(t_{n, i}, t_{n}+s h_{n}\right) e_{h}\left(t_{n}+s h_{n}\right) d s .
\end{aligned}
$$

Then the collocation error on the corresponding subintervals $\sigma_{n}$ has the local Lagrange (Peano) representation

$$
e_{h}\left(t_{n}+v h_{n}\right)=\sum_{j=1}^{m} L_{j}(v) \xi_{n, j}+h_{n}^{m} R_{m, n}(v), \quad v \in(0,1]
$$

where $\xi\left(t_{n, j}\right):=e_{h}\left(t_{n, j}\right)$ and

$$
R_{m, n}(v):=\int_{0}^{1} K_{m}(v, z) y^{m}\left(t_{n}+z h_{n}\right) d z
$$

with

$$
K_{m}(v, z):=\frac{1}{(m-1) !}\left\{(v-z)_{+}^{m-1}-\sum_{k=1}^{m} L_{k}(v)\left(c_{k}-z\right)_{+}^{m-1}\right\}, \quad z \in[0,1] .
$$

The exact solution of (13) can be written in the form

$y\left(t_{0}+v h_{0}\right)=\sum_{(j, k)_{\alpha}} \gamma_{j, k}(\alpha)\left(t_{0}+v h_{0}\right)^{j+k(1-\alpha)}+h_{0}^{m} \bar{Y}_{m, 0}(v ; \alpha), \quad v \in[0,1]$,

with

$$
(j, k)_{\alpha}:=\left\{(j, k): j, k \in \mathrm{N}_{0}, j+k(1-\alpha)<m\right\},
$$


and with obvious adaptation of the meaning of the definition of $Y_{m, 0}(v ; \alpha)$. We rewrite this representation as

$$
\begin{aligned}
y\left(t_{0}+v t_{0}\right)= & \sum_{(j, k)_{\alpha}^{\prime}} \gamma_{j, k}(\alpha) h_{0}^{j+k(1-\alpha)} v^{j+k(1-\alpha)} \\
& +\sum_{(j, k)_{\alpha}^{\prime \prime}} \gamma_{j, k}(\alpha) h_{0}^{j+k(1-\alpha)} v^{j+k(1-\alpha)} \\
& +h_{0}^{m} Y_{m, 0}(v ; \alpha), \quad v \in[0,1]
\end{aligned}
$$

where

$$
(j, k)_{\alpha}^{\prime}:=\left\{(j, k): j+k(1-\alpha) \in \mathrm{N}_{0}, j+k(1-\alpha)<m\right\},
$$

and

$$
(j, k)_{\alpha}^{\prime \prime}:=\left\{(j, k): j+k(1-\alpha) \notin \mathrm{N}_{0}, j+k(1-\alpha)<m\right\} .
$$

With self-explanatory meaning of the coefficients $c_{j, k}(\alpha)$, we thus obtain the local representation

$y\left(t_{0}+v h_{0}\right)=\sum_{j=0}^{m-1} c_{j, 0}(\alpha) v^{j}+h_{0}^{1-\alpha} \Phi_{m, 0}(v ; \alpha)+h_{0}^{m} Y_{m, 0}(v ; \alpha), v \in[0,1]$, with

$$
\Phi_{m, 0}(v ; \alpha):=\sum_{(j, k)_{\alpha}^{\prime \prime}} c_{j, k}(\alpha) v^{j+k(1-\alpha)} .
$$

Suppose now that on $\bar{\sigma}_{0}$ the collocation solution $u_{h} \in S_{m-1}^{(-1)}\left(I_{h}\right)$ is expressed in the form

$$
u_{h}\left(t_{0}+v h_{0}\right)=\sum_{j=0}^{m-1} d_{j, 0} v^{j}, \quad v \in[0,1] .
$$

This allows us to write the collocation error on $\bar{\sigma}_{0}$ as

$$
\begin{aligned}
e_{h}\left(t_{0}+v h_{0}\right)= & \sum_{j=0}^{m-1} \beta_{j, 0}(\alpha) v^{j}+h_{0}^{1-\alpha} \sum_{(j, k)_{\alpha}^{\prime \prime}} c_{j, k}(\alpha) v^{j+k(1-\alpha)} \\
& +h_{0}^{m} R_{m, 0}(v ; \alpha), \quad v \in[0,1]
\end{aligned}
$$

with $\beta_{j, 0}(\alpha):=c_{j, 0}(\alpha)-d_{j, 0}$. 
We now return to the error equation (23) corresponding to $n=0$. It follows from

$$
\begin{aligned}
e_{h}\left(t_{0}+c_{i} h_{0}\right) & =\left(\mathcal{V}_{\alpha} e_{h}\right)\left(t_{0}+c_{i} h_{0}\right) \\
& =h_{0}^{\alpha} \int_{0}^{c_{i}}\left(c_{i}-s\right)^{-\alpha} K\left(t_{0}+c_{i} h_{0}, t_{0}+s h_{0}\right) e_{h}\left(t_{0}+s h_{0}\right) d s
\end{aligned}
$$

that the unknown coefficients $\beta_{j, 0}(\alpha)$ in (26) solve the linear algebraic system

$$
\left[V_{m}-h_{0}^{1-\alpha} B_{0}(\alpha)\right] \beta_{0}(\alpha)=h_{0}^{1-\alpha} \mathbf{q}_{0}(\alpha)+h_{0}^{m} \rho_{0}(\alpha) .
$$

Here, $V_{m} \in L\left(\mathbb{R}^{m}\right)$ denotes the Vandermonde matrix based on the collocation parameters $\left\{c_{i}\right\}$, and the components of the vectors $\mathbf{q}_{0}(\alpha)$ and $\rho_{0}(\alpha)$ can be deduced from (6.2.40). Due to the continuity and the boundedness of the kernel $K$ and the remainder term $R_{m, 0}(\cdot ; \alpha)$ the inverse matrix $\left[V_{m}-h_{0}^{1-\alpha} B_{0}(\alpha)\right]^{-1}$ exists for all $\alpha \in(0,1)$ and is uniformly bounded for sufficiently small $h_{0}$. This in turn implies that, since $m \geq 1$,

$$
\left\|\beta_{0}(\alpha)\right\|_{1} \leq B h_{0}^{1-\alpha} \quad(\alpha \in(0,1))
$$

holds for some constant $B$, and thus

$$
\left|e_{h}\left(t_{0}+v h_{0}\right)\right| \leq\left\|\beta_{0}(\alpha)\right\|_{1}+\gamma_{0}(\alpha) h_{0}^{1-\alpha}+\gamma_{1}(\alpha) h_{0}^{m}, \quad v \in[0,1],
$$

with appropriate constants $\gamma_{0}(\alpha), \gamma_{1}(\alpha)$ and $h_{0} \in(0, \bar{h})$. If the grading exponent $r=r(\alpha)$ is chosen as $r=\mu /(1-\alpha)$, with $1-\alpha \leq \mu \leq m$, then we have

$$
h_{0}^{1-\alpha}=\left(T N^{-r}\right)^{1-\alpha}=T^{1-\alpha} N^{-\mu}=\mathcal{O}\left(h^{\mu}\right) \quad(h:=T / N),
$$

and hence

$$
\left\|e_{h}\right\|_{0, \infty}:=\max _{v \in[0,1]}\left|e_{h}\left(t_{0}+v h_{0}\right)\right|=\mathcal{O}\left(h^{\mu}\right) .
$$

Assume now that $1 \leq n \leq N-1$. It follows from the error equation (23) and the corresponding expression for $\left(\mathcal{V}_{\alpha} e_{h}\right)\left(t_{n, i}\right)$ that

$$
\begin{aligned}
{\left[\mathcal{I}_{m}-h_{n}^{1-\alpha} B_{n}(\alpha)\right] \mathcal{E}_{n}=} & \sum_{\ell=1}^{n-1} h_{\ell}^{1-\alpha} B_{n}^{(\ell)}(\alpha) \mathcal{E}_{\ell}+h_{0}^{1-\alpha} q_{n}^{(0)}(\alpha) \\
& +h_{n}^{m+1-\alpha} \rho_{n}(\alpha)+\sum_{\ell=1}^{n-1} h_{\ell}^{m+1-\alpha} \rho_{n}^{(\ell)}(\alpha)
\end{aligned}
$$


described by the vectors

$$
\begin{aligned}
q_{0}(\alpha) & :=\left(\int_{0}^{1}\left(\frac{t_{n, i}-t_{0}}{h_{0}}-s\right)^{-\alpha}\right. \\
& \left.\cdot K\left(t_{n, i}, t_{0}+s h_{0}\right) e_{h}\left(t_{0}+s h_{0}\right) d s \quad(i=1, \ldots, m)\right)^{T}, \\
\rho_{n}(\alpha) & :=\left(\int_{0}^{c_{i}}\left(c_{i}-s\right)^{-\alpha}\right. \\
\cdot & \left.K\left(t_{n, i}, t_{n}+s h_{n}\right) R_{m, n}(s ; \alpha) d s \quad(i=1, \ldots, m)\right)^{T}, \\
\rho_{n}^{(\ell)}(\alpha) & :=\left(\int_{0}^{1}\left(\frac{t_{n, i}-t_{\ell}}{h_{\ell}}-s\right)^{-\alpha}\right. \\
\cdot & \left.K\left(t_{n, i}, t_{\ell}+s h_{\ell}\right) R_{m, \ell}(s ; \alpha) d s \quad(i=1, \ldots, m)\right)^{T} .
\end{aligned}
$$

As [9, Theorem 6.2.1] showed, $\left[\mathcal{I}_{m}-h_{n}^{1-\alpha} B_{n}(\alpha)\right]^{-1}$ exists and is uniformly bounded whenever $h_{n} \in(0, \bar{h})$ : there is a constant $D_{0}(\alpha)$ so that

$$
\left\|\left(\mathcal{I}_{m}-h_{n}^{1-\alpha} B_{n}(\alpha)\right)^{-1}\right\|_{1} \leq D_{0}(\alpha), \quad(n=1, \ldots, N-1) .
$$

Thus, (28) yields a generalized discrete Gronwall inequality,

$$
\begin{aligned}
\left\|\mathcal{E}_{n}\right\|_{1} \leq & D_{0}(\alpha)\left(\sum_{\ell=1}^{n-1} h_{\ell}^{1-\alpha}\left\|B_{n}^{(\ell)}(\alpha)\right\|_{1}\right. \\
& \cdot\left\|\mathcal{E}_{\ell}\right\|_{1}+h_{0}^{1-\alpha}\left\|q_{n}^{(0)}(\alpha)\right\|_{1}+h_{n}^{m+1-\alpha}\left\|\rho_{n}(\alpha)\right\|_{1} \\
& \left.+\sum_{\ell=1}^{n-1} h_{\ell}^{m+1-\alpha}\left\|\rho_{n}^{(\ell)}(\alpha)\right\|_{1}\right), \quad(n=1, \ldots, N-1) .
\end{aligned}
$$

Using Lemma 5.1, assume now that $\ell<n-1$. In this case, we obtain

$$
\begin{aligned}
\int_{0}^{1}\left(\frac{t_{n, i}-t_{\ell}}{h_{\ell}}-s\right)^{-\alpha} s^{\nu} d s \leq & \int_{0}^{1}\left(\frac{t_{n}-t_{\ell}}{h_{\ell}}-s\right)^{-\alpha} d s=\frac{1}{1-\alpha}\left\{\left(\frac{t_{n}-t_{\ell}}{h_{\ell}}\right)^{1-\alpha}\right. \\
\left.-\left(\frac{t_{n}-t_{\ell}}{h_{\ell}}-1\right)^{1-\alpha}\right\} & =\frac{1}{1-\alpha}\left(\frac{t_{n}-t_{\ell}}{h_{\ell}}\right)^{1-\alpha}
\end{aligned}
$$




$$
\cdot\left\{1-\left[1-\left(\frac{t_{n}-t_{\ell}}{h_{\ell}}\right)^{-1}\right]^{1-\alpha}\right\}
$$

The application of the Mean-Value theorem to the function $f(z):=$ $(1-z)^{1-\alpha}$, with $z:=\left[\left(t_{n}-t_{\ell}\right) / h_{\ell}\right]^{-1}$, leads without difficulty to

$$
\int_{0}^{1}\left(\frac{t_{n, i}-t_{\ell}}{h_{\ell}}-s\right)^{-\alpha} d s \leq\left(\frac{t_{n}-t_{\ell}}{h_{\ell}}\right)^{-\alpha}\left(1-\theta_{n, \ell}\left(\frac{t_{n}-t_{\ell}}{h_{\ell}}\right)^{-1}\right)^{-\alpha}
$$

where $\theta_{n, \ell}$ is some number between 0 and 1 . Since, as pointed out above,

$$
0<h_{0}<h_{1}<\cdots<h_{n-1}<h_{n}<\cdots<h_{N-1}=h,
$$

it follows that

$$
\frac{t_{n}-t_{\ell}}{h_{\ell}}=\frac{h_{n-1}+\cdots+h_{\ell+1}+h_{\ell}}{h_{\ell}} \geq \frac{(n-\ell) h_{\ell}}{h_{\ell}}=n-\ell,
$$

and so

$$
1-\theta_{n, \ell}\left(\frac{t_{n}-t_{\ell}}{h_{\ell}}\right)^{-1} \geq 1-\left(\frac{t_{n}-t_{\ell}}{h_{\ell}}\right)^{-1} \geq 1-\left(\frac{h_{\ell+1}+h_{\ell}}{h_{\ell}}\right)^{-1} \geq \frac{1}{2}
$$

whenever $\ell \leq n-2$.

Recall now the definition of the matrices $B_{n}^{(\ell)}(\alpha)$ and the vectors $\rho_{n}^{(\ell)}(\alpha)(\ell<n)$ from [9, subsection 6.2.1]. It is easy to verify that

$$
\left\|B_{n}^{(\ell)}(\alpha)\right\|_{1} \leq D_{1}(\alpha)(n-\ell)^{-\alpha} \quad(\ell<n)
$$

and

$$
\left\|\rho_{n}^{(\ell)}(\alpha)\right\|_{1} \leq R_{1}(\alpha)(n-\ell)^{-\alpha} \quad(\ell<n) .
$$

with appropriate constants $D_{1}(\alpha)$ and $R_{1}(\alpha)$ depending on $m$ and the bounds for $K$ and the uniform norms of the Lagrange fundamental polynomials $L_{j}$. The inequality (30) now becomes

$$
\begin{aligned}
\left\|\mathcal{E}_{n}\right\|_{1} \leq & \gamma_{0}(\alpha) h^{1-\alpha} \sum_{\ell=1}^{n-1}(n-\ell)^{-\alpha}\left\|\mathcal{E}_{(\ell)}\right\|_{1}+\gamma_{1}(\alpha) h_{0}^{1-\alpha} \\
& +\gamma_{2}(\alpha) h_{n}^{m+1-\alpha}+\gamma_{3}(\alpha) \sum_{\ell=1}^{n-1} h_{\ell}^{m+1-\alpha}(n-\ell)^{-\alpha},
\end{aligned}
$$

with $1 \leq n \leq N-1$ and appropriate constants $\gamma_{i}(\alpha)(i=1,2,3)$. 
Recall the generalized discrete Gronwall inequality and $[\mathbf{9}$, Theorem 6.1.19]: we have $z_{\ell}:=\left\|\mathcal{E}_{(\ell)}\right\|_{1}$, and the sequence $\left\{\gamma_{n}\right\}$ given by

$$
\begin{gathered}
\gamma_{n}:=\gamma_{1}(\alpha) h_{0}^{1-\alpha}+\gamma_{2}(\alpha) h_{n}^{m+1-\alpha}+\gamma_{3}(\alpha) \sum_{\ell=1}^{n-1} h_{\ell}^{m+1-\alpha}(n-\ell)^{-\alpha}, \\
(n \geq 1)
\end{gathered}
$$

is clearly non-decreasing. Moreover, we have

$$
\sum_{\ell=1}^{n-1} h_{\ell}^{1-\alpha}(n-\ell)^{-\alpha} \leq \frac{T^{1-\alpha}}{1-\alpha}, \quad n=1, \ldots, N .
$$

This is easily verified by observing that, for any uniform mesh,

$$
\begin{aligned}
\int_{0}^{t_{n}}\left(t_{n}-s\right)^{-\alpha} d s & =h^{1-\alpha} \sum_{\ell=0}^{n-1} \int_{0}^{1}(n-\ell-s)^{-\alpha} d s \\
& \geq h^{1-\alpha} \sum_{\ell=0}^{n-1}(n-\ell)^{-\alpha}
\end{aligned}
$$

where the last expression represents the lower Riemann sum (left rectangular quadrature approximation) for the given integral whose integrand is convex on $\left[0, t_{n}\right)$.

Hence, we have found a uniform upper bound for $\gamma_{n}$, namely,

$$
\begin{aligned}
\gamma_{n} \leq \bar{\gamma}: & =\gamma_{1}(\alpha) h_{0}^{1-\alpha}+\gamma_{2}(\alpha)+h_{n}^{m+1-\alpha}+\gamma_{3}(\alpha) h^{m} T^{1-\alpha} /(1-\alpha) \\
& =\gamma_{1}(\alpha) h_{0}^{1-\alpha}+\left[\gamma_{2}(\alpha) h_{0}^{1-\alpha}+\gamma_{3}(\alpha) T^{1-\alpha} /(1-\alpha)\right] h^{m} .
\end{aligned}
$$

Combining with (31) leads to

$$
\left\|\mathcal{E}_{n}\right\|_{1} \leq E_{1-\alpha}\left(\gamma_{0}(\alpha) \Gamma(1-\alpha)(n h)^{1-\alpha}\right) \cdot h_{0}^{1-\alpha} \cdot \bar{\gamma} .
$$

By using

$$
n h \leq n r T N^{-1}=(n / N) r T \leq r T, \quad n=1, \ldots, N,
$$

we have

$$
h_{0}^{1-\alpha}=\left(T N^{-r}\right)^{1-\alpha}=T^{1-\alpha} N^{-r(1-\alpha)}=T^{1-\alpha} N^{-\mu}
$$

for any graded $I_{h}$ with grading exponent $r=\mu /(1-\alpha)(1-\alpha \leq \mu \leq m)$. Therefore, $\left\|\mathcal{E}_{n}\right\|_{1} \leq B h^{\mu}(1 \leq n \leq N-1)$, and so, by (24) and (27), we arrive at the desired global estimate for $\left\|e_{h}\right\|_{\infty}$. 


\section{REFERENCES}

1. I. Babuska and P. Chatzipantelidis, On solving elliptic stochastic partial differential equtions, Comp. Meth. Appl. Mech. Eng. 191 (2002), 4093-4122.

2. I. Babuska, K. Liu and R. Tempone, Solving stochastic partial differential equations based on the experimental data, Math. Models Meth. Appl. Sci. 13 (2003), 415-444.

3. I. Babuška, M. Motamed and R. Tempone, A stochastic multiscale method for the elastodynamic wave equation arising from fiber composites, Comp. Meth. Appl. Mech. Eng. 276 (2014), 190-211.

4. I. Babuska, F. Nobile and R. Tempone, A stochastic collocation method for elliptic partial differential equations with random input data, SIAM J. Numer. Anal. 45 (2007), 1005-1034.

5. I. Babuška, F. Nobile and R. Tempone, A stochastic collocation method for elliptic partial differential equations with random input data, SIAM Rev. 52 (2010), 317-355.

6. I. Babuska, R. Tempone and G.E. Zouraris, Galerkin finite element approximations of stochastic elliptic partial differential equations, SIAM J. Numer. Anal. 42 (2004), 800-825.

7. Solving elliptic boundary vualue problems with uncertain coefficients by the finite element method: The stochastic formulation, Comp. Meth. Appl. Mech. Eng. 194 (2005), 1251-1294.

8. A. Bharucha-Reid, Random integral equations, Academic Press, New York, 1972.

9. H. Brunner, Collocation methods for Volterra integral and related functional differential equations, Cambr. Mono. Appl. Comp. Math. 15, Cambridge University Press, Cambridge, 2004.

10. H. Brunner and D. Schötzau, hp-Discontinous Galerkin time-stepping for Volterra integrao-differential equations, SIAM J. Numer. Anal. 44 (2006), 224-245.

11. Y. Cao, T. Herdman and Y. Xu, A hybrid collocation method for Volterra integral equations with weakly singular kernels, SIAM J. Numer. Anal. 41 (2003), $364-381$.

12. Y. Cao and Y. Xu, Singularity preserving Galerkin methods for weakly singular Fredholm integral equations, J. Int. Eq. Appl. 6 (1994), 303-334.

13. Y. Cao, R. Zhang and K. Zhang, Finite element method and discontinuous Galerkin method for stochastic scattering problem of Helmholtz type in $\mathbb{R}^{d}(d=$ 2,3), Potential Anal. 28 (2008), 301-319.

14. A. Chkif, A. Cohen, M. Migloratiz, F. Nobile and R. Tempone, Discrete least squares polynomial approximation with random evaluations-Application to parametric and stochastic elliptic PDEs, Report 2013.

15. H. Elman, O. Ernst, D. Leary and M. Stewart, Efficient iterative algorithms for the stochastic finite element method with application to acoustic scattering, Comp. Meth. Appl. Mech. Eng. 194 (2005), 1037-1055. 
16. G.S. Fishman, Monte Carlo: Concepts, algorithms, and applications, Springer Ser. Op. Res., Springer-Verlag, New York, 1996.

17. N.J. Ford, M. Morgado and M. Rebelo, Nonpolynomial collocation approximation of solutions to fractional differential equations, Fract. Calc. Appl. Anal. 16 (2013), 874-891.

18. P. Frauenfelder, C. Schwab and R.A. Todor, Finite elements for elliptic problems with stochatic coefficients, Comp. Meth. Appl. Mech. Eng. 194 (2005), 205-228.

19. A. Gaudagnini and S. Neumann, Nonlocal and localized analysis of conditional mean steady state flow in bounded, randomly nonuniform domains, 1 . Theory and computational approach, 2. Computational examples, Water Resources Res. 35 (1999), 2999-3039.

20. R.G. Ghanem and P.D. Spanos, Stochastic finite element: A spectral approach, Springer-Verlag, New York, 1991.

21. M. Grigoriu, Stochastic calculus: Applications in science and engineering, Birkhäuser Boston, 2002.

22. M. Khodabin, K. Maleknejad, M. Rostami and M. Nouri, Numerical approach for solving stochastic Volterra-Fredholm integral equations by stochastic operational matrix, Comp. Math. Appl. 64 (2012), 1903-1913.

23. Y.P. Lin, K. Zhang and J. Zou, Studies on some perfectly matched layers for one-dimensional time-dependent systems, Adv. Comp. Math. 30 (2009), 1-35.

24. M. Loéve, Probability theory I, 4th ed., Grad. Text Math. 45, SpringerVerlag, New York, 1977.

25. Probability theory II, 4th ed., Grad. Text Math. 46, Springer-Verlag, New York, 1978.

26. K. Maleknejad, M. Khodabin and M. Rostami, A numerical method for solving $m$-dimensional stochastic Itô-Volterra integral equations by stochastic operational matrix, Comp. Math. Appl. 63 (2012), 133-143.

27. L. Mathelin, M.Y. Hussaini and T.A. Zang, Stochastic approaches to uncertainty quantification in CFD simulations, Num. Algor. 38 (2005), 209-236.

28. H. Matthies and A. Keese, Galerkin methods for linear and nonlinear elliptic stochastic partial differential equations, Comp. Meth. Appl. Mech. Eng. 194 (2005), 209-236.

29. T. Petersdorff and C. Schwab, Sparse finite element methods for operator equations with stochastic data, Appl. Math. 51 (2006), 145-180.

30. A. Quarteroni and A. Valli, Numerical approximation of partial differential equation, Springer Ser. Comp. Math. 23, Springer-Verlag, Berlin, 1994.

31. D. Szynal and S. Wędrychowicz, On solutions of a stochastic integral equation of the Volterra type with applications for chemotherapy, J. Appl. Prob. 25 (1988), 257-267.

32. M.A. Tatang, Direct incorporation of uncertainty in chemical and environmental engineering systems, Ph.D. thesis, MIT, 1995. 
33. R.A. Todor, Sparse perturbation algorithms for ellipitic PDE's with stochastic data, Ph.D. thesis, Dipl. Math., University of Bucharest, 2005.

34. C.P. Tsokos and W.J. Padgett, Random integral equations with applications to stochastic systems, Lect. Notes Math., Springer Verlag, New York, 1971.

35. _ Random integral equations with applications to life sciences and engineering, Academic Press, New York, 1974.

36. N. Wiener, The homogenous chaos, Amer. J. Math. 60 (1938), 897-936.

37. C.L. Winter, D.M. Tartakovsky and A. Guadagnini, Groundwater flow in heterogenous composite aquifer, Water Resource Res. 38 (2002), doi:10.1029/2001WR000450.

38. D. Xiu and J.S. Hesthaven, High-order collocation methods for differential equations with random inputs, SIAM J. Sci. Comp. 27 (2005), 1118-1139.

39. D. Xiu and G.E. Karniadakis, The Wiener-ASkey polynomial chaos for stochastic differential equations, SIAM J. Sci. Comp. 24 (2002), 619-644.

School of Mathematics, Jilin University, Changchun, Jilin, 130023, P.R. China and Department of Mathematics and Statistics, Auburn University, Auburn AL 36849

Email address: yzc0009@auburn.edu

School of Mathematics, Jilin University, Changchun, Jilin, 130023, P.R. CHINA

Email address: zhangran@jlu.edu.cn 